\title{
Rapid identification of Trichoderma koningiopsis and Trichoderma longibrachiatum using sequence characterized amplified region markers
}

\author{
Mohamed M. Hassan ${ }^{1,2^{*}}$ (D, Mona A. Farid ${ }^{3}$ and Ahmed Gaber ${ }^{1,4}$
}

\begin{abstract}
Molecular markers enable the detection and classification of fungi isolated from their natural environments. To develop species-specific markers for detecting Trichoderma koningiopsis and T. Iongibrachiatum, the sequence-characterized amplified region technique, using 20 inter-simple sequence repeat-polymerase chain reaction primers, was performed. The two specific markers for amplifying a single unique band consistent with T. koningiopsis and T. longibrachiatum, which were absent with other Trichoderma strains, were successfully identified. These fragments had no meaningful sequence homology with known sequences available in the National Center for Biotechnology Information and TrichOKEY databases. Compared with traditional identification techniques, these markers can facilitate more rapid and less complicated studies of Trichoderma population dynamics and evaluate their establishment after release into agricultural environments.
\end{abstract}

Keywords: Trichoderma spp., Rapid identification, ITS region sequence, ISSR-PCR, SCAR marker

\section{Background}

Trichoderma, first described over 200 years ago, is a fungal genus including more than 200 species that are found globally in different geographical regions and climate zones (Atanasova et al. 2013). The morphological identification and characterization of Trichoderma was first reported in a monograph by Rifai (1969). Trichoderma spp. are simply recognized in culture medium, in which they produce large numbers of characteristics, small, green, or white conidia, from phialides present on the profusely or mildly branched conidiophores (Hassan et al. 2014).

Numerous molecular techniques have been used for Trichoderma identification to investigate the genetic diversity within the genus. These include restriction fragment length polymorphism (Dodd et al. 2004), random

\footnotetext{
*Correspondence: khyate_99@yahoo.com

1 Department of Biology, Faculty of Science, Taif University, Al-Haweiah,

P.O.Box 888, Taif 21974, Kingdom of Saudi Arabia

2Department of Genetics, Faculty of Agriculture, Menoufiya University, Shibin

Al Kawm, Egypt

Full list of author information is available at the end of the article
}

amplified polymorphic DNA (RAPD) (Hermosa et al. 2001; Parmar et al. 2015a), amplified fragment length polymorphism (Devi et al. 2013), inter-simple sequence repeat (ISSR) (Hassan et al. 2014), microsatellite markers (Naef et al. 2006), repetitive element sequence-based polymerase chain reaction (PCR) (Hassan et al. 2014), and sequence analyses (Druzhinina et al. 2005 and Fahmi et al. 2016).

To improve upon these previous methods, a simple marker system must enable rapid and inexpensive species identification. Sequence-characterized amplified region (SCAR) markers (Parmar et al. 2015b) may represent a solution to the problem of identifying Trichoderma. SCAR markers are superior to RAPD and ISSR markers because they are more specific and usually detect only a single locus. In addition, PCR amplification of SCAR markers is less sensitive to the reaction conditions employed. The markers are more likely to be co-dominant and, therefore, reproducible. Previously, RAPD analysis was used to identify unique PCR products that can be converted to SCAR markers for filamentous fungal species or strains of interest (Parmar et 
al. 2015b). Moreover, Qian et al. (2006) used ISSR-PCR to detect specific fragments among different species, and the conversion of an ISSR fragment into a SCAR marker enabled the researchers to distinguish Sinocalycanthus chinensis from closely related species. SCAR markers have been frequently applied in studying insect taxonomy and population biology. Recently, Yuantian et al. (2015) established a simple and reliable strain diagnostic system for T. harzianum, using a PCR-based technique. They first analyzed $T$. harzianum, using RAPD analysis, to assess its genetic diversity and produce a strain-specific molecular marker. The strain-specific fragment was then converted into a SCAR marker. The ISSR-based SCAR-PCR protocol is superior to existing Aspergillus section Flavi detection systems because of its simplicity and minimal sample handling requirements Priyanka et al. (2014). SCAR markers have also been used to identify different species and biotypes of the Asian gall midge (Behura et al. 1999), a major insect pest of rice, and for the specific identification of six Trypanosoma cruzi lineages (Skoneczny et al. 2015)

Here, developing a species-specific SCAR marker based on the ISSR-PCR technique for the rapid molecular identification of T. harzianum and T. koningiopsis among different Trichoderma species was aimed.

\section{Materials and methods Fungal strains}

The eight Trichoderma isolates (T. harzianum, T. koningiopsis, T. virens, T. atroviride, T. asperellum, T. saturnisporum, and two strains of T. longibrachiatum), used in this study, were isolated from Menoufia and Kafer-Elsheikh Governments, Egypt. The stock cultures of these strains were deep-frozen in glycerol and stored at $-80^{\circ} \mathrm{C}$ until used.

\section{DNA extraction}

Trichoderma mycelia were inoculated into potato dextrose agar broth and cultured for 5 days. The genomic DNA from each Trichoderma strain, using a DNeasy ${ }^{\circ}$ Plant Mini Kit (Qiagen, Hilden, Germany) according to the manufacturer's instructions, was extracted.

\section{Molecular identification}

Strain identification was based on the internal transcribed spacer 1 and 2 (ITS1 and ITS2) regions of the ribosomal RNA gene cluster sequences according to the method described by Hassan et al. (2014). All sequences were obtained by PCR and direct amplicon sequencing from the 5' and 3' ends, using a 3130 Genetic Analyzer (Applied Biosystems, Waltham, MA, USA) in the Scientific Research Center of the Biotechnology and Genetic Engineering Unit at Taif University, Saudi Arabia. The sequencing data were compared with the GenBank database (http://
www.ncbi.nlm.nih.gov/BLAST/), using the nucleotide BLAST program to identify homology between the PCR fragments and sequences in the GenBank database. The sequences were deposited in the National Center for Biotechnology Information (NCBI) GenBank database under accession numbers KY214240, KY214241, KY214242, KY 214243, KY214244, KY214245, KY214246, and KY214247. The eight strains were then been used for PCR-based species-specificity evaluation of the markers.

\section{ISSR analysis}

ISSR-PCR, as previously described by Hassan et al. (2014), was performed. Twenty primers for PCR (obtained from Macrogen, Seoul, Korea) were used. The sequences of the ISSR primers were as follows: $(\mathrm{GA})_{8} \mathrm{~T},(\mathrm{GA})_{8} \mathrm{~A},(\mathrm{AG})_{8} \mathrm{TG}$, $(\mathrm{GA})_{8} \mathrm{TT},(\mathrm{GA})_{8} \mathrm{TC},(\mathrm{AC})_{8} \mathrm{TT}, \mathrm{C}(\mathrm{GA})_{7} \mathrm{G}, \mathrm{GAC}(\mathrm{GA})_{7}, \mathrm{TAG}$ $(\mathrm{CA})_{7}, \mathrm{CAG}(\mathrm{CA})_{7},(\mathrm{AG})_{8} \mathrm{~T},(\mathrm{AG})_{8} \mathrm{C},(\mathrm{AG})_{8} \mathrm{G},(\mathrm{GA})_{8} \mathrm{C}$, $(\mathrm{CA})_{8} \mathrm{~A},(\mathrm{TC})_{8} \mathrm{C},(\mathrm{AC})_{8} \mathrm{C},(\mathrm{AC})_{8} \mathrm{G},(\mathrm{AG})_{8} \mathrm{TT}$, and $(\mathrm{GA})_{8} \mathrm{~A}$ T. ISSR-PCR was performed in tubes containing $1 \mu \mathrm{l}(20$ ng) of genomic DNA, $12.5 \mu \mathrm{l}$ of GoTaq ${ }^{\circ}$ Green Master Mix (Promega, Madison, WI, USA), $1 \mu \mathrm{l}$ of each primer (20 pmol), and deionized distilled water (up to a total volume of $25 \mu \mathrm{l})$. For DNA amplification, the tube contents were heated at $94{ }^{\circ} \mathrm{C}$ for $10 \mathrm{~min}$ in a C1000 Touch $^{\mathrm{mm}}$ Thermal Cycler (Bio-Rad, Munich, Germany). Subsequently, Taq polymerase was added, followed by 35 cycles of $1 \mathrm{~min}$ at $94^{\circ} \mathrm{C}, 1.5 \mathrm{~min}$ at $52^{\circ} \mathrm{C}$, and $2.5 \mathrm{~min}$ at $72^{\circ} \mathrm{C}$, with a final 7 -min extension at $72{ }^{\circ} \mathrm{C}$. Amplified DNA products were analyzed by electrophoresis in $2 \%$ agarose gels run in Tris/ borate/EDTA buffer. The gels were stained with ethidium bromide $\left(5 \mu \mathrm{g} \cdot \mathrm{ml}^{-1}\right)$. The $100 \mathrm{bp}$ DNA Ladder RTU (GeneDireX ${ }^{\bullet}$, München, Germany) was used as a standard. DNA was visualized by UV illumination, and then photographed, using a Bio-Rad Gel Doc 2000.

\section{Sequencing of strain-specific ISSR amplicons}

Amplified DNA fragments were separated on 1.5\% agarose gels. The selected diagnostic ISSR markers for Trichoderma spp. were excised from the gels, and the DNA fragments were recovered using a QIAquick ${ }^{\circ}$ Gel Extraction Kit (Qiagen), according to the manufacturer's protocol. An aliquot of each recovered DNA fragment was re-amplified, using the corresponding primer to verify that only a single band had been excised. The ISSR fragments were sequenced directly, using the Gene Analyzer 3130 sequencer with the original ISSR primers specific for each amplicon.

\section{SCAR primers and PCR conditions}

The nucleotide sequences of the ISSR fragments were used to design pairs of SCAR primers, which were then synthesized by Macrogen (Korea). PCR amplification was performed with the SCAR primer pairs and the PCR mixtures for the SCAR assays, using the reaction 
Table 1 The sequences producing significant alignments among Trichoderma species

\begin{tabular}{|c|c|c|c|c|}
\hline \multirow[t]{2}{*}{ Trichoderma strains } & \multicolumn{4}{|c|}{ Sequences producing significant alignments } \\
\hline & Accession no. & Description & Accession no. & Score similarity (\%) \\
\hline T. harzianum-1 & KY214240 & T. harzianum- SC50578 & KU896375.1 & 100 \\
\hline T. koningiopsis-2 & KY214241 & T. koningiopsis-KP3 & KP340272.1 & 100 \\
\hline T. virens-3 & KY214242 & T. virens-AXM50550 & KU896371.1 & 100 \\
\hline T. atroviride-4 & KY214243 & T. atroviride 112,086 & AF194020.1 & 92 \\
\hline T. asperellum-5 & KY214244 & T. asperellum ZWPBG7 & KR868322.1 & 99 \\
\hline T. saturnisporm-6 & KY214245 & T. saturnisporm T61 & KC884818.1 & 100 \\
\hline T. longibrachiatum-7 & KY214246 & T. longibrachiatum T-24 & KU317870.1 & 99 \\
\hline T. longibrachiatum-8 & KY214247 & T. longibrachiatum T-24 & KU317870.1 & 99 \\
\hline
\end{tabular}

conditions described above in the ISSR analysis section. All reactions with the SCAR primers were repeated at least three times with suitable controls. The PCR products were visualized in $1.5 \%$ agarose gels stained with ethidium bromide.

\section{Specificity and sensitivity test of the SCAR markers}

The specificities of the SCAR primer pairs were tested in PCR assays according to Devi et al. (2013) against the eight strains of Trichoderma. The sensitivities of the devised SCAR assays were tested by PCR with variable

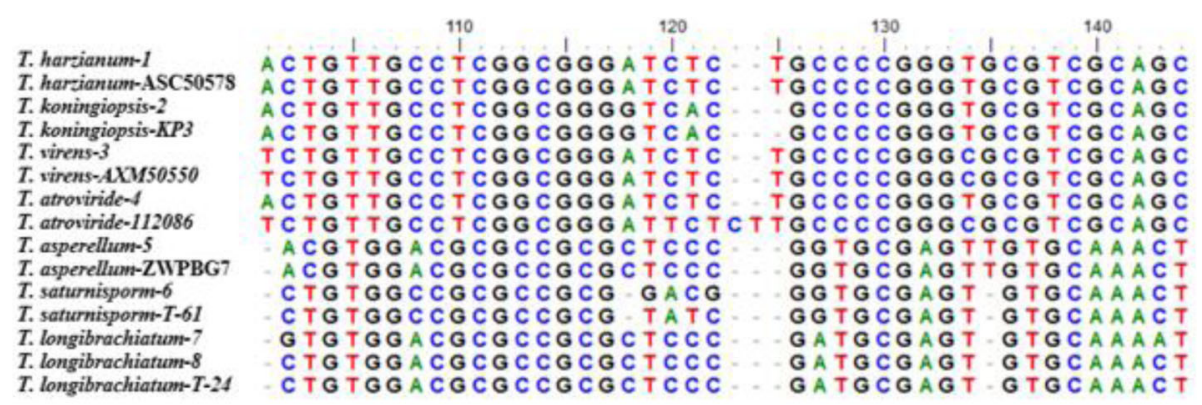

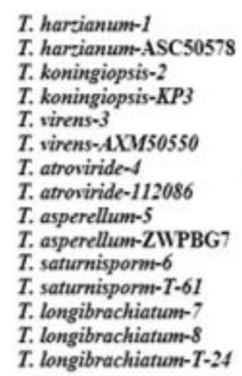

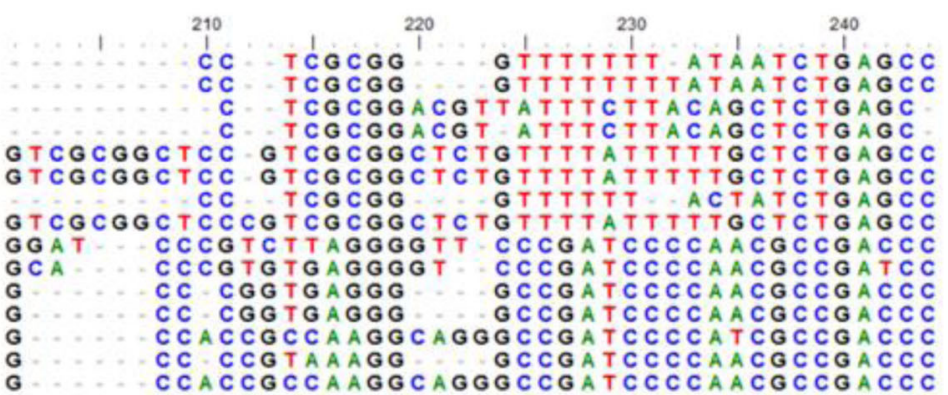

T. hartianum-1

T. hartianum-ASC50578 T. koningiopsis-2

T. koningiopsis-KP3

T. vinens-3

T. virens- 4 XY50550

T. virentride-4

T. atrowirided 12086

T. asperellum-5

T. asperellum-ZWPBG7

T. saturnisporm-6

T. saturnisporm- -61

T. longibrachiatum-?

T. longibrachiatum- 8

T. longibnachiatum- $T-24$

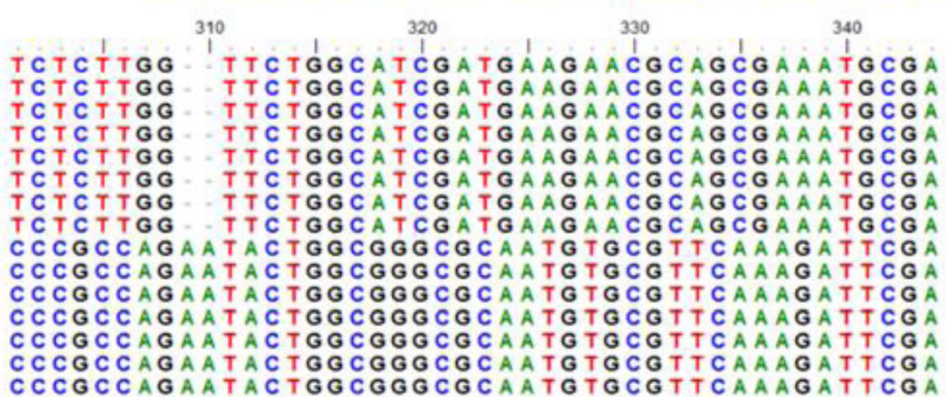

Fig. 1 Multiple alignment of the ITS nucleotide sequences of eight Trichoderma strains. The alignment was achieved among eight Trichoderma strains and related sequences of some Trichoderma strains in the GenBank database, using the BioEdit program 
quantities of genomic DNA. The sensitivity assays were replicated at least twice.

\section{Results and discussion}

Sequencing of ITS regions

Initially, the ITS region sequences of the eight Trichoderma spp., which were morphologically identified at the Genetics Department in the Faculty of Agriculture at Menoufia University, Egypt, as described by Rifai (1969) were confirmed. The ITS regions were amplified in different strains, resulting in PCR products of 550 to 650 base pairs (bp), which were sequenced directly. The sequences were subjected to BLAST analysis against the NCBI database. The strains were identified as $T$. harzianum, $T$. koningiopsis, T. virens, T. atroviride, T. asperellum, $T$. saturnisporum, and two strains of $T$. longibrachiatum. Species of the Trichoderma genus are typically found as soil-borne or wood-decaying fungi. Some species are economically important because they produce industrial enzymes (cellulase and hemicellulase) and antibiotics or act as biocontrol agents (Hassan 2014 and Fahmi et al. 2016). Therefore, correct identification of Trichoderma at the species level is important. Morphological identification of species has proven unreliable (Sharma et al. 2009 and Shahid et al. 2013) because of their high degrees of similarity. In addition, identification based on host preferences and morphological differences is not reliable due to their sensitivities to environmental factors; thus, molecular methods have been introduced recently for accurate identification.
Molecular patterns in genomic DNA are hypothetically not affected by these variations and can be useful for species identification and the resolution of doubtful cases (Gajera and Vakharia 2010 and Błaszczyk et al. 2011).

\section{Multiple alignment of the ITS region nucleotide sequences in different Trichoderma strains}

The ITS sequences were subjected to multiple alignment, using the online tool BioEdit (http://www.mbio.ncsu.edu/ BioEdit/page2.html). Both intra- and inter-species variations were observed in the ITS sequences. Among the eight strains of Trichoderma spp., the ITS region nucleotide sequences showed $98.6 \%$ similarity. When the sequences were aligned by the database sequences, they showed $99-100 \%$ similarity at all strains, except for the $T$. atroviride-4 sequence, which shared (92\%) similarity with the strain T. atroviride 112086 (accession number AF194020.1; Table 1). However, greater differences between the other strains and the two T. longibrachiatum (7 and 8) strains were observed. As well, 99\% homology was observed between the two $T$. longibrachiatum strains tested and the published T. longibrachiatum-T24 strain. The positions of the ITS sequences, including their variations among the strains, are shown in Fig. 1.

\section{Phylogenetic analysis of the ITS sequences}

A phylogenetic tree with the nucleotide sequences of the cloned ITS regions of the eight Trichoderma strains, using MEGA 5.1 software, was constructed. The strains

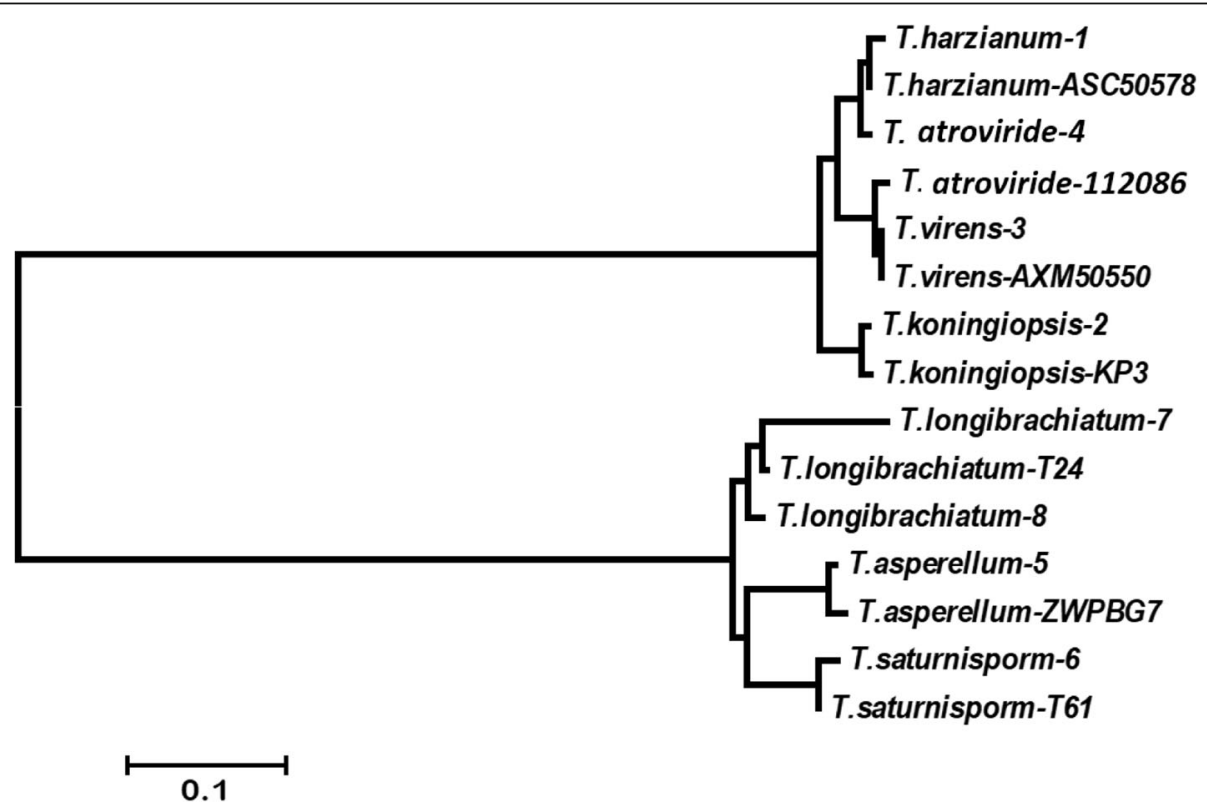

Fig. 2 Phylogenetic tree of the eight Trichoderma strains. The tree was built using the nucleotide sequences of the ITS region among the eight strains of the Trichoderma genus used in this study versus other Trichoderma strains deposited in the GenBank database 

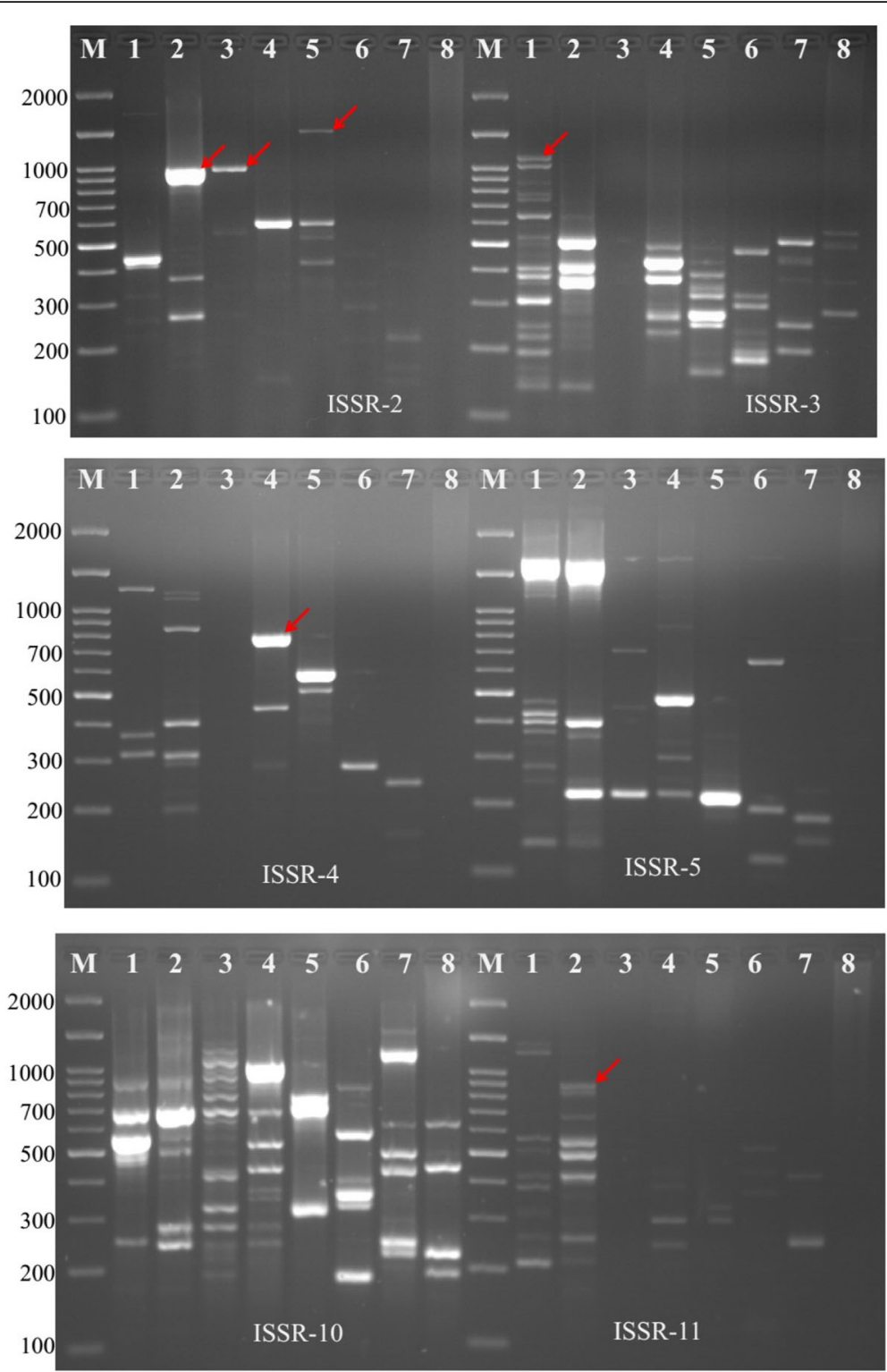

Fig. 3 ISSR-PCR profile of the eight Trichoderma strains using ISSR-2, ISSR-3, ISSR-4, ISSR-5, ISSR-10, and ISSR-11 primers. The amplification of DNA from the different species of Trichoderma was used for detecting the polymorphic bands among the isolates. The red arrows showed the speciesspecific bands in some Trichoderma spp. Lane 1, 100 bp DNA ladder

separated into six distinct phylogenetic sub-clades (Fig. 2). Group 1 included isolates of T. harzianum-1, T. harzianum- SC50578, and T. atroviride-4; group 2 included T. atroviride-112086, T. virens-3, and T. virens-AXM50550; group 3 included T. koningiopsis-2 and T. koningiopsis-KP3; group 4 included T. longibrachiatum-7, T. longibrachiatum-8, and T. longibrachiatum-T24; group 5 included T. asperellum-5 and T. asperellum ZWPBG7; and group 6 included T. saturnisporum- 6 and $T$. saturnisporum-T61. Joint phylogenetic analysis of the nucleotide sequences of all ITS regions revealed a marked phylogenetic distance of approximately $100 \%$. The details of the likelihood similarities among the isolates are reflected in the similarity index data (Table 1). The phylogenetic tree that was constructed using the ITS sequences distinguished T. longibrachiatum, T. harzianum, and $T$. atroviride from the other strains. Furthermore, the ITS sequence analysis of $T$. atroviride revealed variations between T. atroviride-4 and T. atroviride-112086; thus, the phylogenetic tree clustered the $T$. atroviride- 4 strains in separate clades. Accordingly, the ITS analysis clearly separated the seven species from each other. Similarly, Kuhls et al. (1997) used sequence analysis to differentiate between $T$. reesei and T. longibrachiatum. Many other researchers have used ITS sequences to identify Trichoderma spp. (Rifai 1969 and Fahmi et al. 2016). 

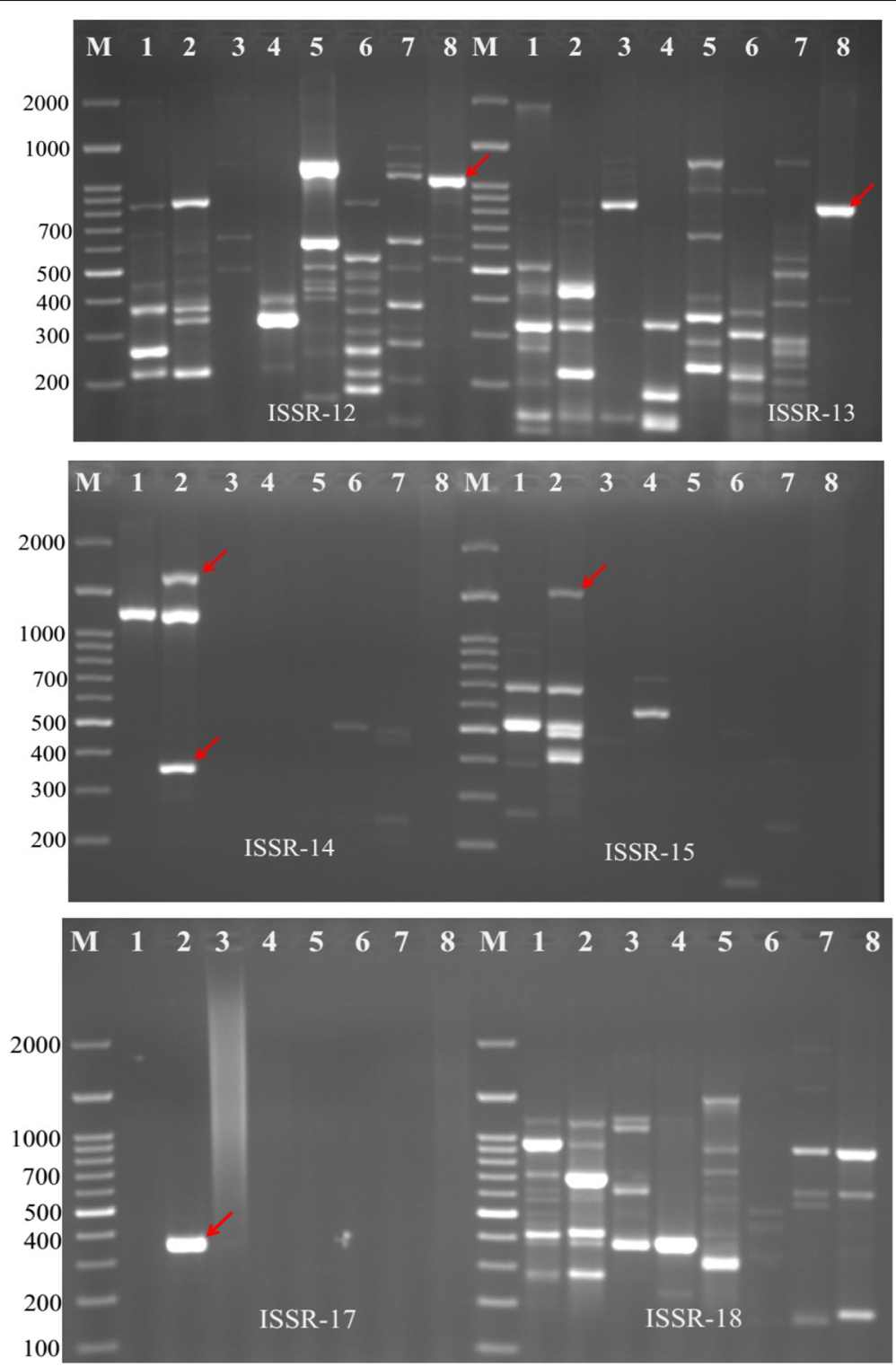

Fig. 4 ISSR-PCR profile of the eight Trichoderma strains using ISSR-12, ISSR-13, ISSR-14, ISSR-15, ISSR-17, and ISSR-18 primers. The amplification of DNA from the different species of Trichoderma was used for detecting the polymorphic bands among the isolates. The red arrows showed the species-specific bands in some Trichoderma spp. Lane 1, 100 bp DNA ladder

Multiple alignment of the ITS sequences of all seven Trichoderma species identified nucleotide sequence differences for differentiating these species. In the multiple alignment, both ends of the ITS region (i.e., the ITS-1 and ITS-2 regions) showed nucleotide sequence variations. Perfect homology was observed in the nucleotide sequences of the $5.8 \mathrm{~S}$ ribosomal DNA gene region. The strains of the Trichoderma spp., that were confirmed by ITS in this study, were included for ISSR analysis. This technique utilizes oligonucleotide primers to generate amplification products that can be used to assess genetic variations within the entire genome, rather than variations within a single genetic region. This characteristic of ISSR analysis has been exploited by researchers investigating genetic relatedness in several fungal species, often to the extent of differentiating races. The ISSR analysis of eight strains belonging to seven different species showed both intra- and inter-species differences.

\section{ISSR profiling of the Trichoderma strains}

ISSR analysis on the eight Trichoderma spp. that were confirmed by ITS sequence homology was performed. Initially, the DNA of the different strains belonging to each species was screened, using 20 ISSR primers. Only 
Table 2 List of SCAR primers name and sequence with related Trichoderma strains

\begin{tabular}{llll}
\hline $\begin{array}{lll}\text { No. } \\
\begin{array}{l}\text { Strain } \\
\text { code }\end{array}\end{array}$ & $\begin{array}{l}\text { SCAR primers } \\
\text { name }\end{array}$ & Sequence $\left(5^{\prime} \rightarrow 3^{\prime}\right)$ \\
\hline 1 & T2 & $\begin{array}{l}\text { SCAR }-2-\mathrm{F} \\
\text { SCAR }-2-\mathrm{R}\end{array}$ & $\begin{array}{l}\text { CGC TGA TCG TTC GCC TTC AAT } \\
\text { AAT AAC GAT TAT CCG CGC GAC } \\
\end{array}$ \\
& & C \\
2 & T8 & SCAR $-12-\mathrm{F}$ & ATG CAG TGA TGC TTA GCC C \\
& SCAR $-12-\mathrm{R}$ & GAA GTC AGT GAT GCA GTG \\
\hline
\end{tabular}

T2 T. koningiopsis, T8 T. longibrachiatum

18 of the 20 primers produced polymorphic bands (Fig. 3). Those 18 primers were used to study the polymorphisms in the individual strains of the different species. Of the 18 ISSR primers, 15 (ISSR-2, $-3,-4,-5$, $9,-10,-11,-12,-13,-14,-15,-17,-18,-19$, and -20) generated bright, consistent bands unique to particular species (Fig. 3). These unique bands were purified, and the samples were sent for sequencing. ISSR analysis also showed intra-species variations between the two strains of $T$. longibrachiatum. Although ISSR analysis detects differences at the strain level, it may not be effective for rapid and reliable distinction of different of Trichoderma species. Therefore, the ISSR markers into SCAR markers were converted. Furthermore, ISSR has certain disadvantages, such as inconsistency and the lack of reproducibility in independent laboratories. Speciesspecific markers of fungal taxa must be reliable and capable of reproducibly amplifying genomic sequences.

\section{Determination and validation of the SCAR markers}

Among the 15 ISSR primers that generated unique bands, only 3 (ISSR-2, ISSR-12, and ISSR-13) were selected to design SCAR primers because of the high molecular weight and sharpness bands. ISSR-2 generated polymorphic fragments of 900, 1000, and $1500 \mathrm{bp}$ in T. koningiopsis, $T$. virens, and $T$. asperellum, respectively (Fig. 3). ISSR-12 and ISSR-13 generated unique 900- and 800-bp fragments, respectively, with the $T$. longibrachiatum strains, and no amplification was observed with the other species (Fig. 4). The unique fragments detected for each species were not present in the other species. The fragments produced by the ISSR- 2 and ISSR-12 primers were sent for sequencing as purified PCR products. The sequences from T. koningiopsis, T. virens, T. asperellum, and T. longibrachiatum were analyzed by performing BLAST searches at NCBI and TrichOKEY (http://www.isth.info/tools/ molkey/); however, none of the sequences showed similarity with the Trichoderma sequences in either database. Next, the specific SCAR primers were designed to amplify unique fragments, approximately $500 \mathrm{bp}$ in length, for each marker (Table 2). The putative species-specific SCAR primer pairs, named SCAR-2 and SCAR-12, were used to amplify the genomic DNA of the eight Trichoderma spp.

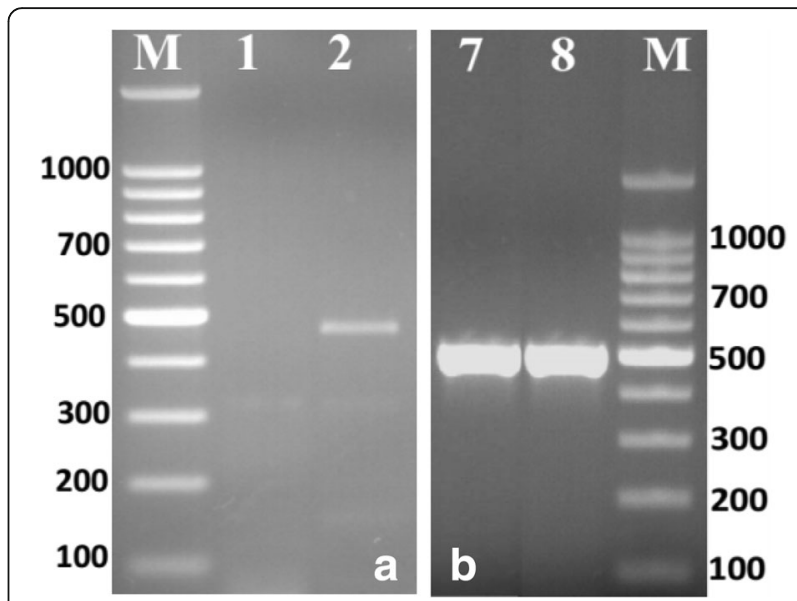

Fig. 5 Validation of the SCAR-2 and SCAR-12 primers against $T$. koningiopsis-2 (a) and T. longibrachiatum-7 and 8 (b)

The SCAR-2 and SCAR-12 primers produced only amplicons of 480 and $510 \mathrm{bp}$, respectively, using T. koningiopsis and T. longibrachiatum as the DNA template (Fig. 5). No amplification was observed for the other species. Obtained results raised doubts regarding the use of ITS sequences alone for the correct identification of Trichoderma species. Shahid et al. (2014) also reported that ITS regions were not universally applicable as species-level markers and that defining the species solely based on the ITS identity may be error-prone, barring $100 \%$ sequence similarity between a strain and the ex-type strain (Lakhani et al. 2016). In this investigation, two species-specific SCAR primers, namely SCAR-2 for T. koningiopsis and SCAR-12 for T. longibrachiatum, were developed from sequenced, putatively species-specific ISSR bands. Therefore, these SCAR markers can be used to rapidly identify new isolates or mixtures of isolates.

\section{Conclusion}

DNA-based methods that provide valuable taxonomic information for Trichoderma are currently being used for identification and phylogenetic classification. Most Trichoderma spp. are morphologically identical and, therefore, were considered as a single species for many years. In this study, stable SCAR markers were developed to identify genotypes within Trichoderma species by amplifying a single, genetically defined locus, using a pair of specific oligonucleotide primers for T. koningiopsis and T. longibrachiatum. Different DNA-based markers, such as amplified fragment length polymorphism, SSR, ISSR, and RAPD, can be used to produce these markers. Thus, the high dependability of SCAR markers could result in replacing RAPD and other DNA-based methods, which are costly and time-consuming. 


\section{Acknowledgements}

Not applicable for this study.

\section{Funding}

Not applicable' for this study.

\section{Availability of data and materials}

The data and material used during the current study are available from the corresponding author on reasonable request.

\section{Authors' contributions}

This study was conceived and designed by both authors, MMH and MF. The laboratory work was carried out by $\mathrm{MMH}$. Data were analyzed by MF and AG. The manuscript was written by MMH and MF. The manuscript was revised by AG. All authors have accepted the final version of the manuscript.

\section{Ethics approval and consent to participate}

Ethical approval and consent to participate are not required for this study.

\section{Consent for publication}

Not applicable

\section{Competing interests}

The authors declare that they have no competing interests.

\section{Publisher's Note}

Springer Nature remains neutral with regard to jurisdictional claims in published maps and institutional affiliations.

\section{Author details}

'Department of Biology, Faculty of Science, Taif University, Al-Haweiah, P.O.Box 888, Taif 21974, Kingdom of Saudi Arabia. ${ }^{2}$ Department of Genetics, Faculty of Agriculture, Menoufiya University, Shibin Al Kawm, Egypt. ${ }^{3}$ Genetics Department, Faculty of Agriculture, Kafrelsheikh University, Kafr El-Sheikh, Egypt. ${ }^{4}$ Department of Genetics, Faculty of Agriculture, Cairo University, Giza, Egypt.

Received: 4 September 2018 Accepted: 15 February 2019

Published online: 05 March 2019

\section{References}

Atanasova L, Druzhinina IS, Jaklitsch WM (2013) Two hundred Trichoderma species recognized on the basis of molecular phylogeny. In: Mukherjee PK, Horwitz BA, Singh US, Mukherjee M, Schmoll M (eds) Trichoderma: biology and applications. CABI, Wallingford, pp 10-42

Behura SK, Sahu SC, Rajamani S (1999) Differentiation of Asian rice gall midge, Orseolia oryzae (Wood-Mason), biotypes by sequence characterized amplified regions (SCARS). Insect Mol Biol 8(3):391-397

Błaszczyk L, Popiel D, Chełkowski J (2011) Species diversity of Trichoderma in Poland. J Appl Genet 52(2):233-243

Devi TP, Prabhakaran N, Kamil D (2013) Development of SCAR marker for specific detection of Aspergillus flavus. Afr J Microbiol Res 7(9):783-790

Dodd SL, Hill RA, Steward A (2004) Monitoring the survival and spread of the biocontrol fungus Trichoderma atroviride (C65) on kiwifruit using a molecular marker. Aust Plant Pathol 33(2):189-196

Druzhinina IS, Kopchinskiy AG, Komoń M (2005) An oligonucleotide barcode for species identification in Trichoderma and Hypocrea. Fungal Genet Biol 42(10): 813-828

Fahmi Al, Eissa RA, El-Halfawi KA (2016) Identification of Trichoderma spp. by DNA barcode and screening for cellulolytic activity. J Microb Biochem Technol 8(3):202-209

Gajera HP, Vakharia DN (2010) Molecular and biochemical characterization of Trichoderma isolates inhibiting a phytopathogenic fungi Aspergillus niger Van Tieghem. Physiol Mol Plant Pathol 74:274-282

Hassan MM (2014) Influence of protoplast fusion between two Trichoderma spp. on extracellular enzymes production and antagonistic activity. Biotechnol Biotechnol Equip 28(6):1014-1023

Hassan MM, Gaber A, El-Hallous El (2014) Molecular and morphological characterization of Trichoderma harzianum from different Egyptian soils. Wulfenia 21:80-96
Hermosa MR, Grondona I, Díaz-Mínguez JM (2001) Development of a strainspecific SCAR marker for the detection of Trichoderma atroviride 11, a biological control agent against soil-borne fungal plant pathogens. Curr Genet 38(6):343-350

Kuhls K, Lieckfeldt E, Samuels GJ (1997) Revision of Trichoderma section Longibrachiatum including related teleomorphs based on an analysis of ribosomal DNA internal transcribed spacer sequences. Mycologia 89:442-460

Lakhani HN, Vakharia DN, Hassan MM (2016) Fingerprinting and molecular comparison among two parental strains of Trichoderma spp. and their corresponding fusants produced by protoplast fusion. Biotechnol Biotechnol Equip 30(6):1065-1074

Naef A, Senatore M, Défago G (2006) A microsatellite based method for quantification of fungi in decomposing plant material elucidates the role of Fusarium graminearum DON production in the saprophytic competition with Trichoderma atroviride in maize tissue microcosms. FEMS Microbiol Ecol 55(2): 211-220

Parmar HJ, Hassan MM, Bodar NP (2015a) In vitro antagonism between phytopathologic fungi Sclerotium rolfsii and Trichoderma strains. Int J Appl Sci Biotechnol 3(1):16-19

Parmar HJ, Hassan MM, Bodar NP (2015b) Development of SCAR marker for specific detection of Trichoderma harzanium and Trichoderma viride. Amer J Microbiol Res 3(1):45-49

Priyanka SR, Uppalapati SR, Kingston JJ (2014) Development of ISSR-derived SCAR marker-targeted PCR for identification of Aspergillus section Flavi members. Lett Appl Microbiol 58(5):414-442

Qian Y, Ying-xiong Q, Yan-qi Q (2006) Species-specific SCAR markers for authentication of Sinocalycanthus chinensis. J Zhejiang Univ Sci B 7(11):868-872

Rifai MA (1969) Revision of the genus Trichoderma. Mycol Pap 116:1-56

Shahid M, Srivastava M, Kumar V (2014) Phylogenetic diversity analysis of Trichoderma species based on internal transcribed spacer (ITS) marker. Afr J Biotechnol 13(3):449-455

Shahid M, Srivastava M, Sharma A (2013) Morphological, molecular identification and SSR marker analysis of a potential strain of Trichoderma/Hypocrea for production of a bioformulation. J. Plant. Pathol Microbiol 4(10):204-210

Sharma K, Mishra AK, Misra RS (2009) Morphological, biochemical and molecular characterization of Trichoderma harzianum isolates for their efficacy as biocontrol agents. J Phytopathol 157(1):51-56

Skoneczny D, Oskiera M, Szczech M (2015) Genetic diversity of Trichoderma atroviride strains collected in Poland and identification of loci useful in detection of within-species diversity. Folia Microbiol 60(4):297-307

Yuantian S, Libo T, Zhenyu S (2015) Development of RAPD based SCAR marker for rapid identification of Trichoderma harzianum. Plant Dis Pests 6(2):12-15

\section{Submit your manuscript to a SpringerOpen ${ }^{\circ}$ journal and benefit from:}

- Convenient online submission

- Rigorous peer review

- Open access: articles freely available online

High visibility within the field

- Retaining the copyright to your article

Submit your next manuscript at $>$ springeropen.com 